\title{
Not Just Complicated Cholecystitis-Beware of Risk Factors!
}

\author{
Begoña Peinado Iribar, Estibaliz Alvarez Peña, and Ines Rubio-Perez
}

\begin{abstract}
Background: The association between individual risk factors and a higher incidence of multi-drug-resistant infections may transform a common surgical condition such as accute cholecystitis into a life-threatening situation. Case Presentation: A 75-year-old male with multiple comorbidities including active chemotherapy presented to the emergency department with fever and acute upper right quadrant pain. He presented low blood pressure but no other systemic inflammatory response syndrome (SIRS) parameters. Resuscitation with intravenous fluids and a first dose of antibiotics was prescribed, with new blood tests ordered for the next morning. The patient was re-evaluated by the next day's team and his clinical condition and blood results had worsened, so he was taken to the operating room. A cholecystectomy was performed, abdominal fluid was sent for culture, and empirical antibiotic (meropenem) was prescribed for his severe condition. Cultures were positive for extended spectrum $\beta$-lactamase (ESBL) Escherichia coli and Klebsiella pneumoniae, adequately treated empirically. The patient remained in the intensive care unit for 48 hours but the rest of his post-operative course was uneventful and he was discharged a week later.

Conclusions: We report a case of complicated cholecystitis, a common condition in the emergency department, but in a patient with multiple risk factors and infection with a multi-resistant pathogen, that can lead to a lifethreatening situation if an early source control and adequate antibiotic therapy are not performed immediately.
\end{abstract}

Keywords: health-care-associated infections; multi-drug-resistant infections; risk factors; severe cholecystitis

A CUTE CHOLECYSTITIS is a common surgical condition and treatment can be vary depending on the clinical characteristics of the patient. Normally it is a mild-to-moderate infection that can be treated by antibiotic therapy, cholecystectomy, or even percutaneous drainage in elderly and comorbid patients. There are various guidelines and consensus, such as the Tokyo Guidelines, that propose algorithms for treatment selection depending on clinical presentation [1]. Nevertheless, there are some cases in which the patient may have associated risk factors such as diabetes mellitus or immunosuppression [2] that can increase the risk of progression to a gangrenous cholecystitis faster and with a poor symptomatic repercussion. Also, in oncologic patients with frequent visits to the hospital, health-careassociated infections must be considered, and the risk of atypical and multi-drug-resistant bacteria should not be overlooked when prescribing empirical therapy [3].

Keeping all this in mind, the aim of this case report is to demonstrate how a common condition such as acute cholecystitis must be considered in some cases as a severe infection, knowing that sometimes the typical clinical signs may not be present or may be misleading. Concomitant medication and impaired immunity can affect clinical signs and symptoms, and usual sepsis criteria such as sequential organ failure assessment (SOFA) or systemic inflammatory response syndrome (SIRS) [4] may not be enough to determine severity. A thorough medical history considering all risk factors, a detailed examination, and rapid diagnosis and treatment are imperative to prevent fatal outcomes.

\section{Case Presentation}

A 75-year-old male presented to the emergency department with fever (temperature, $38^{\circ} \mathrm{C}$ ), diarrhea, and right upper quadrant abdominal pain that had worsened in the last four days. He had a medical history of high blood pressure, diabetes mellitus, dyslipidemia, dilated myocardiopathy, and angina and was being treated with $\beta$-blockers. He had undergone neoadjuvant therapy and a low anterior resection for rectal cancer five months prior and was currently receiving chemotherapy (Xeloda ${ }^{\circledR}$, Genentech, South San Francisco,

General Surgery Department. Hospital Universitario La Paz, Madrid, Spain.

(c) Begoña Peinado Iribar et al. 2017; Published by Mary Ann Liebert, Inc. This Open Access article is distributed under the terms of the Creative Commons License (http://creativecommons.org/licenses/by/4.0), which permits unrestricted use, distribution, and reproduction in any medium, provided the original work is properly credited. 


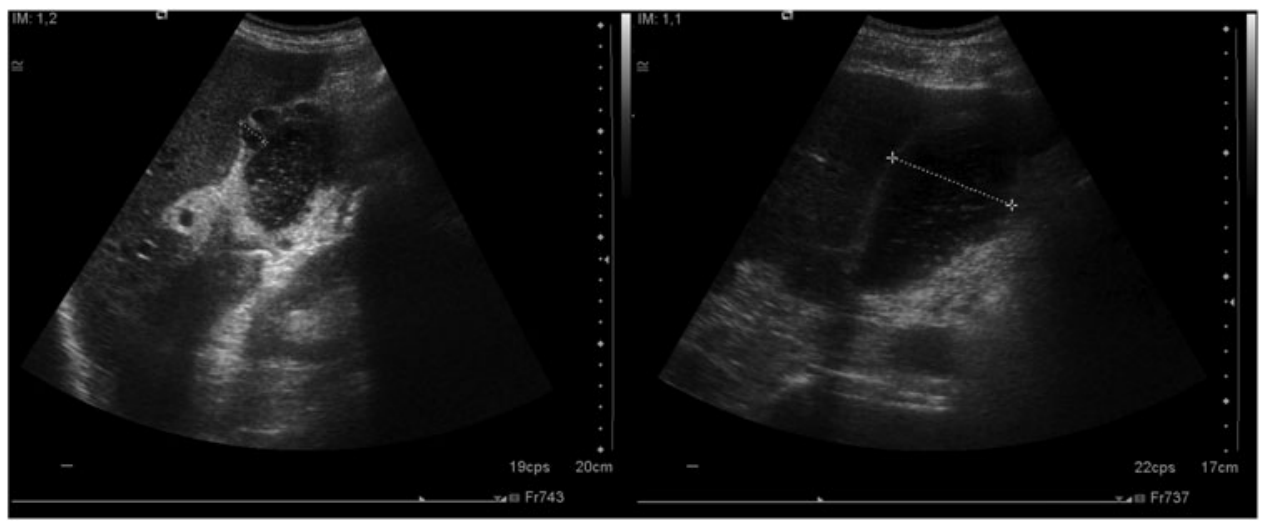

FIG. 1. Ultrasound showing distended gallbladder, floating intra-luminal echogenicities, thickening of the gallbladder wall (with micro-abscesses), and peri-cholecystic fluid.

CA). The patient had received the last dose of chemotherapy one week ago. Upon arrival, his blood pressure was $64 / 33 \mathrm{~mm}$ $\mathrm{Hg}$, basal oxygen saturation was $99 \%$, and his heart rate was 75 beats per minute. On examination of the abdomen, he presented tenderness and a positive Murphy sign. No mass was palpable and there were no signs of peritoneal irritation. He described no other symptoms, but referred to being recently treated with ciprofloxacin for a non-complicated urinary tract infection by his general practitioner.

The patient was evaluated by the medical team and examined for fever and hypotension as an oncologic patient. Blood tests and cultures were taken and chest radiograph performed. He had a high C-reactive protein $(242 \mathrm{mg} / \mathrm{L})$, no leucocytosis, and a high neutrophil count $(81 \%)$. Lactate levels were $2.3 \mathrm{mmol} / \mathrm{L}$. The rest of the blood laboratory analyses were normal: creatinine and urea presented normal values, as well as liver function and bilirubin. Abdominal radiography revealed normal gas distribution and chest radiograph showed no alterations. A surgical consult was made by the emergency department team, and after evaluating the patient and receiving results of abdominal ultrasound, he was diagnosed with acute cholecystitis. Ultrasound of the right upper quadrant showed a distended gallbladder with many floating intra-luminal echogenicities and thickening of the gallbladder wall with micro-abscesses and a small peri-cholecystic fluid collection (Fig. 1). Resucitation with intravenous fluids and a first dose of empirical ertapenem were prescribed, with new blood tests ordered for the following day.

The patient was re-evaluated the next day and his clinical condition and blood results had worsened. His pain had increased and his blood pressure was $90 / 55 \mathrm{~mm} \mathrm{Hg}$; the surgical team decided to perform emergency surgery. Because of previous surgical scars and septic condition, an open approach was undertaken. A gangrenous cholecystitis with localized peritonitis was identified in the operating room (Fig. 2). A cholecystectomy and abdominal lavage were performed and a drain placed in the sub-hepatic area. Abdominal fluid was sent for culture, and empirical meropenem was prescribed for his severe condition. He stayed in the intensive care unit (ICU) for 48 hours and received a blood transfusion, but the rest of his post-operative course was uneventful; he was discharged after completing seven days of antibiotic treatment. Cultures were positive for extended spectrum $\beta$-lactamase (ESBL) Escherichia coli and Klebsiella pneumoniae (both resistant to penicillin and cephalosporins but sensitive to carbapenems) adequately treated empirically (Table 1).

\section{Discussion}

It is important to consider the individual risk factors for a complicated infection in each patient. Diabetes mellitus, cancer, immunosuppression, previous antibiotic therapy, or hospitalization are all risk factors for multi-drug-resistant bacteria, so these must all be considered when prescribing empirical therapy [5]. In our case, the patient had multiple risk factors upon admission but severity criteria were misleading. As a result of the treatment with $\beta$-blockers he did not present tachycardia. Perhaps secondary to his immunosuppression and chemotherapy he did not have an elevated white blood cell

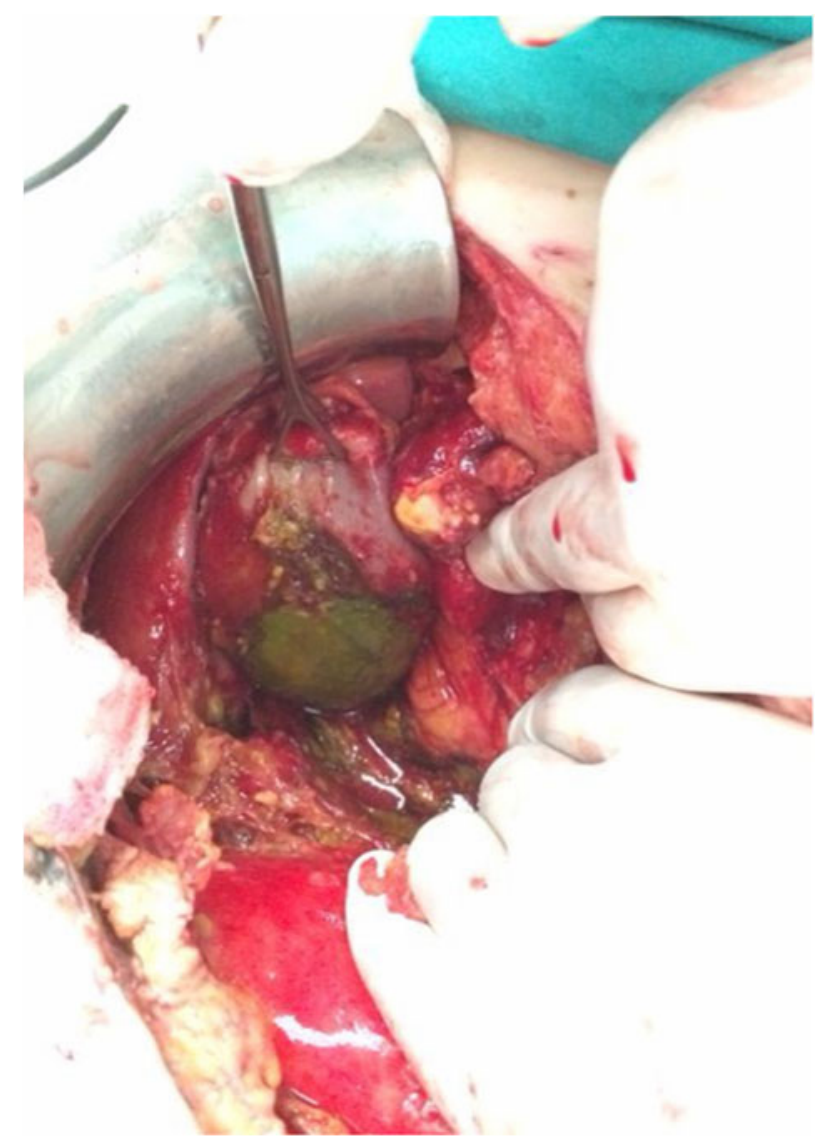

FIG. 2. Intra-operative findings: gangrenous cholecystitis with localized peritonitis. 
Table 1. Bile Cultures Results, Showing ESBL-Producing E. COLI AND K. PNEUMONIAE And Their Antibiotic Sensitivity Profile

\section{AEROBES}

(1) Klebsiella pneumoniae

(2) Escherichia Coli

(1)

\begin{tabular}{lllll}
\hline Ampicilin & $\mathrm{R}$ & $>16$ & $\mathrm{R}$ & $>16$ \\
Amoxicilin-Clavulanate & $\mathrm{S}$ & $<=8 / 14$ & $\mathrm{~S}$ & $<=8 / 14$ \\
Piperacilin-Tazobactam & $\mathrm{S}$ & $<=8 / 14$ & $\mathrm{~S}$ & $<=8 / 14$ \\
Cefalotin & $\mathrm{R}$ & $>16$ & $\mathrm{R}$ & $>16$ \\
Cefuroxime & $\mathrm{R}$ & $>16$ & $\mathrm{R}$ & $>16$ \\
Cefotaxime & $\mathrm{R}$ & 32 & $\mathrm{R}$ & 32 \\
Ceftazidime & $\mathrm{R}$ & 16 & $\mathrm{R}$ & 16 \\
Cefepime & $\mathrm{S}$ & $<=1$ & $\mathrm{R}$ & 8 \\
Aztreonam & $\mathrm{R}$ & 8 & $\mathrm{R}$ & 8 \\
Ertapenem & $\mathrm{S}$ & $<=0.5$ & $\mathrm{~S}$ & $<=0.5$ \\
Imipenem & $\mathrm{S}$ & $<=1$ & $\mathrm{~S}$ & $<=1$ \\
Colistin & $\mathrm{S}$ & $<=4$ & $\mathrm{~S}$ & $<=4$ \\
Gentamicin & $\mathrm{S}$ & $<=2$ & $\mathrm{~S}$ & $<=2$ \\
Tobramycin & $\mathrm{S}$ & $<=2$ & $\mathrm{~S}$ & $<=2$ \\
Amikacin & $\mathrm{S}$ & $<=8$ & $\mathrm{~S}$ & $<=8$ \\
Tigeciclin & $\mathrm{S}$ & $<=1$ & $\mathrm{~S}$ & $<=1$ \\
Ciprofloxacin & $\mathrm{S}$ & $<=0.5$ & $\mathrm{~S}$ & $<=0.5$ \\
Cotrimoxazole & $\mathrm{S}$ & $<=2 / 38$ & $\mathrm{~S}$ & $<=2 / 38$ \\
\hline
\end{tabular}

MIC $\mathrm{mcg} / \mathrm{ml}$ breakpoints according to EUCAST 2013

ANAEROBES

Sterile

count. His blood pressure was low upon admission but responded to fluids initially and was considered to be influenced by the previous diarrhea. He was not tachypneic nor had an altered mentation, so systemic inflammatory response syndrome (SIRS) or quick sequential organ failure assessment score (qSOFA) criteria would not be alarming initially [4]. However, these patients can be on the verge of immune collapse and can progress rapidly to severe sepsis, so we have to reevaluate their clinical condition continuously and suspect any infectious source, find it as early as possible, and initiate antibiotic therapy. This case is a reminder always to check for early markers of severity being aware of these types of confounders.

Another interesting issue is to consider the group of patients presenting with many risk factors, usual contact with the hospital (day care, dialysis, etc.), and a higher incidence of multidrug-resistant infections that has been classified as health-careassociated. These must be considered, even in cases of cholecystitis, appendicitis, or other abdominal infections apparently arising from the community setting, because the selection of the empirical therapy may vary thus influencing the outcome [6].

An adequate and early source control is essential to ensure the best possible outcome [5]. It is important to administer antibiotic therapy early enough but also to recognize when a patient is septic and may need a surgical intervention. In this case, perhaps the surgical team should have considered an operation immediately, performing an early source control and thus avoiding the progression to sepsis because this can increase morbidity and mortality.

Intra-operative cultures are important to tailor the antibiotic treatment and confirm appropriate spectrum. The recent emer- gence of multi-drug-resistant organisms such as communityaquired methicillin-resistant Staphylococcus aureus, ESBL producing Enterobacteriaceae, carbapenemase-producing Klebsiella pneumoniae and vancomycin-resistant Enterococcus faecium, make it seem reasonable to recommend culturing bile [3].

\section{Conclusion}

In conclusion, any infection, as simple as it may seem, can cause sepsis and shock if not treated appropriately and aggressively from the beginning, so we must be aware of this and help trainees recognize risk factors and signs and symptoms of severity.

\section{Acknowledgments}

The authors would like to thank the Surgical Infection Society Europe for the opportunity of participating in the educational SIS-E Olympics in Hamburg (June 2017).

\section{Author Disclosure Statement}

No competing financial interests exist.

\section{References}

1. Takada T, Strasberg SM, Solomkin JS, et al. TG13: Updated Tokyo Guidelines for the management of acute cholangitis and cholecystitis. J Hepatobiliary Pancreat Sci 2013;20:1-7.

2. Cho JY, Han HS, Yoon YS et al. Risk factors for acute cholecystitis and a complicated clinical course in patients with symptomatic cholelithiasis. Arch Surg 2010;145:329-333.

3. Perez F, Adachi J, Bonomo RA. Antibiotic-resistant gramnegative bacterial infections in patients with cancer. Clin Infect Dis 2014;59(Suppl 5):S335-S339.

4. Singer M, Deutschman CS, Seymour CW, et al. The Third International Consensus Definitions for Sepsis and Septic Shock (Sepsis-3). JAMA 2016;315:801-810.

5. Montravers P, Blot S, Dimopoulos G, et al. Therapeutic management of peritonitis: A comprehensive guide for intensivists. Intensive Care Med 2016;42:1234-1247.

6. Chen YH, Hsueh PR. Changing bacteriology of abdominal and surgical sepsis. Curr Opin Infect Dis 2012;25:590-595.

Address correspondence to: Dr. Begoña Peinado Iribar C/ Comandante Zorita $N^{\circ} 19$ $4^{\circ}$ B. 28020. Madrid Spain

E-mail: bpiribar@gmail.com

$\begin{aligned} & \text { Abbreviations Used } \\ & \text { ESBL }=\text { extended spectrum } \beta \text {-lactamase } \\ & \text { ICU }=\text { intensive care unit } \\ & \text { SIRS }=\text { systemic inflammatory response syndrome } \\ & \text { qSOFA }=\text { quick sequential organ failure assessment } \\ & \text { SOFA }=\text { sequential organ failure assessment }\end{aligned}$

Cite this article as: Peinado Iribar B, Alvarez Peña E, Rubio-Perez I (2017) Not just a complicated cholecystitis-Beware of risk factors! Surgical Infections Case Reports 2:1, 98-100, DOI: 10.1089/crsi.2017.0029 